\title{
Silencing c-Jun inhibits autophagy and abrogates radioresistance in nasopharyngeal carcinoma by activating the PI3K/AKT/mTOR pathway
}

\author{
Yongchu Sun ${ }^{1}$, Kaihua Chen ${ }^{1}$, Guoxiang Lin $^{1}$, Fangzhu Wan ${ }^{1}$, Li Chen ${ }^{1}$, Xiaodong Zhu ${ }^{1,2,3}$ \\ ${ }^{1}$ Department of Radiation Oncology, Guangxi Medical University Cancer Hospital, Nanning, China; ${ }^{2}$ Guangxi Key Laboratory of Early Prevention \\ and Treatment for Regional High Frequency Tumor, Guangxi Medical University, Nanning, China; ${ }^{3}$ Department of Oncology, Affiliated Wuming \\ Hospital of Guangxi Medical University, Nanning, China \\ Contributions: (I) Conception and design: X Zhu; (II) Administrative support: K Chen; (III) Provision of study materials or patients: G Lin; (IV) \\ Collection and assembly of data: F Wan, L Chen; (V) Data analysis and interpretation: Y Sun; (VI) Manuscript writing: All authors; (VII) Final \\ approval of manuscript: All authors. \\ Correspondence to: Xiaodong Zhu. Department of Radiation Oncology, Guangxi Medical University Cancer Hospital, No. 71 He Di Road, Nanning \\ 530021, China. Email: zhuxdonggxmu@126.com.
}

Background: Radioresistance plays an important role in the failure of radiotherapy (RT) for nasopharyngeal
carcinoma (NPC), leading to poor prognosis. The purpose of this study was to explore the relationship between
the expression of the c-Jun oncogene and the prognosis of NPC. In addition, we investigated the potential
mechanisms of c-Jun in the regulation of tumor growth and radioresistance in NPC.
Methods: c-Jun expression in NPC tissues and nasopharyngeal mucosa tissues was evaluated using
immunochemistry. c-Jun and its downstream targets were verified by dual-luciferase reporter assays.
Inhibitors or activators were used to interfere with the PI3K/AKT/mTOR pathway. Protein expression was
analyzed by western blotting. NPC nude mouse xenograft models were used to investigate the potential
effects of c-Jun and ionizing radiation in vivo.

Results: The expression of c-Jun in NPC tissues was significantly higher than that in normal nasopharyngeal mucosa (NNM) tissues, and Cox regression analysis revealed that c-Jun overexpression was an independent risk factor for poor prognosis in NPC patients. Both in vitro and in vivo experiments verified that c-Jun targeted PI3K/AKT signaling. We also performed an in vivo study showing that c-Jun knockdown effectively suppressed NPC growth in a xenograft tumor model by autophagy inhibition, and these effects were accompanied by the upregulation of p-PI3K p-AKT, p-mTOR, and P62 and downregulation of LC3-II expression.

Conclusions: High expression of c-Jun was correlated with poor prognosis in NPC patients. c-Jun knockdown increased cell sensitivity to radiation by inhibiting autophagy activation via the PI3K/AKT/ mTOR signaling pathway. The present study provides a theoretical basis for a promising treatment for radioresistant NPC by inhibiting c-Jun expression.

Keywords: c-Jun; nasopharyngeal carcinoma (NPC); PI3K/AKT/mTOR; autophagy; radioresistance

Submitted May 10, 2021. Accepted for publication Jun 29, 2021.

doi: $10.21037 / \mathrm{atm}-21-2563$

View this article at: https://dx.doi.org/10.21037/atm-21-2563

\section{Introduction}

Nasopharyngeal carcinoma (NPC) has a high incidence of head and neck malignant tumors in southern China and some countries in Southeast Asia (1). Due to the special anatomical location of NPC and the pathological nature of the tumor, the current standard treatment for patients 
with early-stage disease is radiotherapy (RT) or concurrent radiochemotherapy for those with advanced-stage disease. With the rapid advancement of technology radiobiological knowledge, better treatment outcomes have been achieved. Nevertheless, $15-30 \%$ of patients with NPC still suffer local recurrence and distant metastasis after standardized treatment (2), in which radioresistance has emerged as an essential factor. The mechanisms of radioresistance in NPC remain elusive (3). Therefore, deciphering new molecular targets and pathways of radioresistance is of great significance to enhance the radiosensitivity and prognosis of NPC.

c-Jun is a member of the AP-1 transcription factor family, a dimeric complex including Jun (c-Jun, JunB and JunD), Fos (c-Fos, FosB, Fra-1 and Fra-2) (4). c-Jun is closely related to cell growth and functional metabolism (5) and plays a regulatory role in cell proliferation, apoptosis, tumor metastasis, and invasion (6). c-Jun can activate Beclin-1, upregulate the expression of LC3-II, and increase autophagy and cell apoptosis (7). It has been shown that c-Jun overexpression mediates autophagy in hepatocellular carcinoma and prostate cancer $(8,9)$, and suppression of c-Jun contributes to a reduction in autophagy and an increase in apoptosis.

Autophagy is a cell-protection mechanism initiated under nutrient depletion (10). Autophagy can also prevent tumor cells from undergoing apoptosis under metabolic stress, thereby promoting the growth of tumors (11). Therefore, autophagy inhibition may be a new strategy for the treatment of tumors. Intriguingly, Digomann et al. indicated that autophagy inhibition is an effective way to increase the sensitivity of head and neck squamous cell carcinomas to RT (12). A growing body of evidence has demonstrated that high expression of c-Jun can upregulate autophagy $(13,14)$. In addition, mammalian target of rapamycin (mTOR) is a key node in the autophagy-related pathway, whose activation can inhibit autophagy and promote the apoptosis of tumor cells, and the PI3K/AKT/ mTOR pathway is a classic pathway of autophagy, affecting the proliferation and chemosensitivity of NPC (15). In a previous study, biomarkers related to the radioresistance of the radioresistant cell line CNE-2R and its parental cell line CNE-2, which were screened by gene chip and protein interaction network diagrams, showed that c-Jun was highly expressed in CNE-2R cells relative to CNE-2, and inhibition of c-Jun expression in CNE-2R cells could increase the radiosensitivity of CNE-2R cells (11). We tentatively propose that c-Jun affects autophagy by regulating the $\mathrm{PI} 3 \mathrm{~K} / \mathrm{AKT} / \mathrm{mTOR}$ signaling pathway, thereby participating in the occurrence of RT resistance in NPC.

This study explored the relationship between c-Jun expression and the prognosis of NPC. Our results represent the first evidence of its kind indicating that the activation of c-Jun is involved in radioresistance through upregulation of autophagy. We further investigated whether c-Jun is involved in autophagy through the $\mathrm{PI} 3 \mathrm{~K} / \mathrm{AKT} / \mathrm{mTOR}$ pathway. We hope our findings can contribute to building a foundation for the treatment of radioresistant NPC.

We present the following article in accordance with the REMARK reporting checklist (available at https://dx.doi. org/10.21037/atm-21-2563).

\section{Methods}

\section{Patients}

Paraffin-embedded tissue specimens were collected from 69 patients who received intensity-modulated RT (IMRT) at the Guangxi Medical University Cancer Hospital from January 2013 to October 2014. NPC tissue specimens were obtained by nasopharyngoscopy biopsy surgery. The inclusion criteria were as follows: (I) patients initially diagnosed with NPC by biopsy; (II) no evidence of distant metastasis from imaging manifestations; (III) patients without chemotherapy, RT, or biotherapy for NPC; (IV) no medical history of other tumor diseases; (V) patients who were not pregnant or breastfeeding; (VI) patients with Karnofsky score $\geq 80$ points; and (VII) patients who received IMRT. In addition, all patients underwent assessment of disease stage according to the Union for International Cancer Control (UICC) tumor-node-metastasis (TNM) classification (16). Normal nasopharyngeal mucosa (NNM) tissues of 21 noncancer patients were collected as the control group. All procedures performed in this study involving human participants were in accordance with the Declaration of Helsinki (as revised in 2013). The study was approved by Research Ethics Committee of Guangxi Medical University Cancer Hospital and informed consent was taken from all the patients.

\section{Follow-up}

After RT treatment was completed, the patients were followed up every 3 months for the first year, every 6 months in the second and third years, and then once a year thereafter. 
The median follow-up period was 45.21 (ranging from 3.09 to 62 ) months.

\section{Immunobistochemistry (IHC)}

IHC was determined by the streptavidin-peroxidase (SP) method. The NPC tissue sections were heated in a $60{ }^{\circ} \mathrm{C}$ thermostat, deparaffinized by xylene, fully rehydrated by different gradient concentrations of alcohol, placed in citric acid-sodium citrate buffer ( $\mathrm{pH}$ 6.0), and boiled for antigen retrieval. The slides were blocked with endogenous peroxidase at room temperature and then incubated with primary antibody (Cell Signaling Technology, Danvers, MA, USA) at $4{ }^{\circ} \mathrm{C}$ overnight. Then, biotin-labeled secondary antibody working fluid and SP were added. Images were visualized by following a standard method using 3,3'-diaminobenzidine (DAB) as a substrate. In the negative control (NC), primary antibody was omitted. The IHC-stained sections were evaluated by two independent pathologists who were blinded to the clinicopathological data. The staining intensity was scored at 4 levels: 0 for negative staining, 1 for weak staining, 2 for moderate staining, and 3 for strong staining. The percentage score of stained cells was 0 for no staining, 1 for $<30 \%, 2$ for $30-60 \%$, and 3 for $>60 \%$. The total staining score (range, 0-6) for each tissue sample was the sum of the above scores. A score of 0-3 was considered low expression, and a score of 4-6 was classified as high expression.

\section{Cell culture and transfection}

The CNE-2 cell line was purchased from the Experimental Center of Shanghai Cancer Hospital and maintained in our lab. The radioresistant cell line CNE-2R was constructed with fractional irradiation as described in our previous study (17) and preserved by the Experimental Research Department of Guangxi Medical University Cancer Hospital. Cell culture was conducted in RPMI 1640 (Gibco, Thermo Fisher Scientific Inc., Waltham, MA, USA) medium containing $10 \%$ fetal bovine serum (FBS; Gibco), $100 \mathrm{U} / \mathrm{mL}$ penicillin, and $0.1 \mathrm{mg} / \mathrm{mL}$ streptomycin and cultured at $37{ }^{\circ} \mathrm{C}$ and $5 \% \mathrm{CO}_{2}$ in saturated humidity.

Lentiviral infection was performed as described previously (18). CNE-2R cells in logarithmic growth phase were selected, digested and counted, seeded in a sixwell plate $\left(2 \times 10^{5}\right.$ cells/well $)$, and transfected when the cell fusion rate reached approximately $30 \%$. Short hairpin RNAs (shRNAs) were designed by GeneChem (Shanghai,
China): c-Jun lentivirus (c-Jun-shRNA: CAAACCTCAGCAACTTCAA) and NC interference sequence (NC: TCTCCGAACGTGTCACGT). The lentiviral vectors were transfected into CNE-2R cells with a multiplicity of infection (MOI) of 30 in the presence of polybrene and cultured for 96 hours. The efficiency of infection was observed by inverted fluorescence microscopy.

\section{Inbibition of the PI3K/AKT and mTOR signaling patbways}

The mTOR inhibitor rapamycin (RAPA) or the PI3K inhibitor LY294002 was transfected into CNE-2R cells. LY294002 or RAPA was used to downregulate the expression of PI3K and mTOR. To investigate whether PI3K/AKT could regulate mTOR, LY294002 and RAPA were purchased from Sigma-Aldrich (St. Louis, MO, USA), and CNE-2R cells were cultured in RPMI 1640 medium (Gibco) with $10 \%$ FBS containing LY294002 $(20 \mu \mathrm{M})$ or RAPA $(50 \mathrm{nM})$. Protein from the two groups was collected after 6 hours for further western blotting experiments.

\section{Target prediction}

The bioinformatics website Sabiosciences was used to predict whether c-Jun has binding sites with AKT, PI3K or mTOR.

\section{Luciferase reporter assay}

Reporter genes, including the promoter AKT1 (42409-1) and promoter PIK3CG (42408-1), were constructed by GeneChem (Shanghai, China), and CNE-2R cells were cotransfected with luciferase reporters and the c-Jun mimic. Luciferase activity was detected using the Dual Luciferase Assay (Promega, Wisconsin, USA) after 48 hours according to the manufacturer's instructions. The cotransfected cells were fully lysed in 24-well culture dishes containing passive lysis buffer at $4{ }^{\circ} \mathrm{C}$ and then shaken for 3-5 minutes. The firefly luminescence value was detected using a plate Instrument Microplate System (Perkin Elmer, Waltham Mass, USA) immediately after oscillation mixing, and the transfection efficiencies were normalized according to the Renilla activity.

\section{Western blotting assay}

Cells in the logarithmic phase of growth were collected. 
After full lysis with lysis buffer, protein loading buffer was added and denatured at $100{ }^{\circ} \mathrm{C}$ for 5 minutes. Fifty micrograms of each histone was transferred to a polyvinylidene fluoride (PVDF) membrane after SDSPAGE separation. The PVDF membrane was blocked with skimmed milk for 1.5 hours and then incubated with the primary antibodies against c-Jun $(1: 1,000$; CST), AKT, p-AKT (1:1,000; CST), PI3K, p-PI3K (1:1,000; CST), mTOR, p-mTOR (1:1,000; CST), GAPDH (1:1,000; CST), LC3-II (1:1,000; Abcam, Oregon, USA), and P62 (1:1,000; Abcam) in a shaker at $4{ }^{\circ} \mathrm{C}$ overnight. After washing with tris-buffered saline with Tween20 (TBST), the membrane was incubated with a secondary immunoglobin G (IgG) horseradish peroxidase (HRP) antibody (CST) for 1 hour. Enhanced chemiluminescence (ECL, Millipore, Bedford, MA, USA) was used to visualize the protein bands, and the intensity of the protein bands was normalized to GAPDH. Images were obtained by chemiluminescence (Bio-Rad, Hercules, CA, USA).

\section{Xenograft tumor model in nude mice}

Four- to five-week-old male BALB/C nude mice were purchased from Chongqing Tengxinbier Experimental Animal Sales Co., Ltd. The nude mice were randomly divided into six groups and fed in a special pathogen-free (SPF) environment. A $200 \mu \mathrm{L}$ cell suspension $\left(1 \times 10^{7} / \mathrm{mL}\right)$ was injected into the right inguinal area. The tumor sizes of nude mice were observed every 3 days after inoculation, and the long diameter (a) and short diameter (b) of the tumors were measured with an electronic Vernier caliper according to the following tumor volume calculation formula: $\mathrm{V}=$ $1 / 2 \times a \times b^{2}$. When the tumor volume of the transplanted nude mice reached $80-120 \mathrm{~mm}^{3}$ (14 days after inoculation), irradiation was conducted with a source-to-surface distance (SSD) of $100 \mathrm{~cm}$ from the skin margin with an electron linear accelerator (Precise1120, Elekta Instrument AB, Stockholm, Sweden). The mice were fixed on a treatment bed, and tumors in the right groin were located in the center of the irradiation field under 4\% chloral hydrate anesthesia and covered with equivalent membrane. The transplanted tumors were irradiated at a dose of 5 Gy/exposure with $6 \mathrm{MV} \mathrm{X}$-rays once daily on day 14 and 21, with a total dose of $10 \mathrm{~Gy}$.

Transfection of pathway activator or inhibitor into nude mice was then performed. After the first irradiation, the nude mice were intraperitoneally injected with PI3K activator insulin-like growth factor 1 (IGF-1) at a dose of $10 \mu \mathrm{g} / \mathrm{kg}$, PI3K inhibitor LY294002 at a dose of $50 \mathrm{mg} / \mathrm{kg}$, and mTOR inhibitor RAPA at a dose of $1.5 \mathrm{mg} / \mathrm{kg}$. Mice were injected every other day and sacrificed 16 days after the first irradiation. Experiments were performed under a project license granted by the Laboratory Animal Care and Use Committee of Guangxi Medical University Cancer Hospital, in compliance with Guangxi Medical University Cancer Hospital guidelines for the care and use of animals.

\section{Statistical analysis}

Statistical analysis was performed by GraphPad Prism 7.0 software and SPSS Statistics software 23 (IBM Corp., Armonk, NY, USA). All experimental data are presented as the mean $\pm \mathrm{SD}(\bar{x} \pm s)$, and intragroup comparisons were assessed by a two-tailed Student's $t$-test or one-way analysis of variance (ANOVA). Survival curves were obtained by the Kaplan-Meier method and compared by the logrank test. Univariate and multivariate survival analyses were performed for all parameters using Cox proportional hazard regression models. The Spearman rank correlation coefficient was used to determine the correlation between two parameters. All statistical tests were two-tailed, and differences with a $\mathrm{P}$ value $<0.05$ were considered statistically significant.

\section{Results}

\section{Overexpression of c-Fun indicated poor prognosis in NPC patients}

To identify the potential role of c-Jun in determining the clinical outcomes of NPC patients, we assessed its expression in a tissue microarray of 69 NPC patients. IHC results showed that c-Jun was mainly localized to the nucleus (Figure 1A). Hyperchromatic nuclei could be observed occasionally in NNM with vigorous proliferation. As shown in Table 1, positive c-Jun expression was found in 37 of $69(53.6 \%)$ NPC tissues, while it was found in only 4 of $21 \mathrm{NNM}$ tissues. c-Jun expression was obviously increased in NPC tissue compared with NNM tissue (53.6\% vs. $19 \% ; \mathrm{P}<0.01)$. The patient characteristics of the two groups are shown in Table 2. c-Jun expression presented no significant correlation with the age or sex of NPC patients. The results demonstrated that NPC patients with high c-Jun expression had shorter overall survival than did those with negative expression of c-Jun (Figure 1B). Univariate Cox regression analysis showed that positive c-Jun expression was correlated with shorter overall survival of NPC 
A

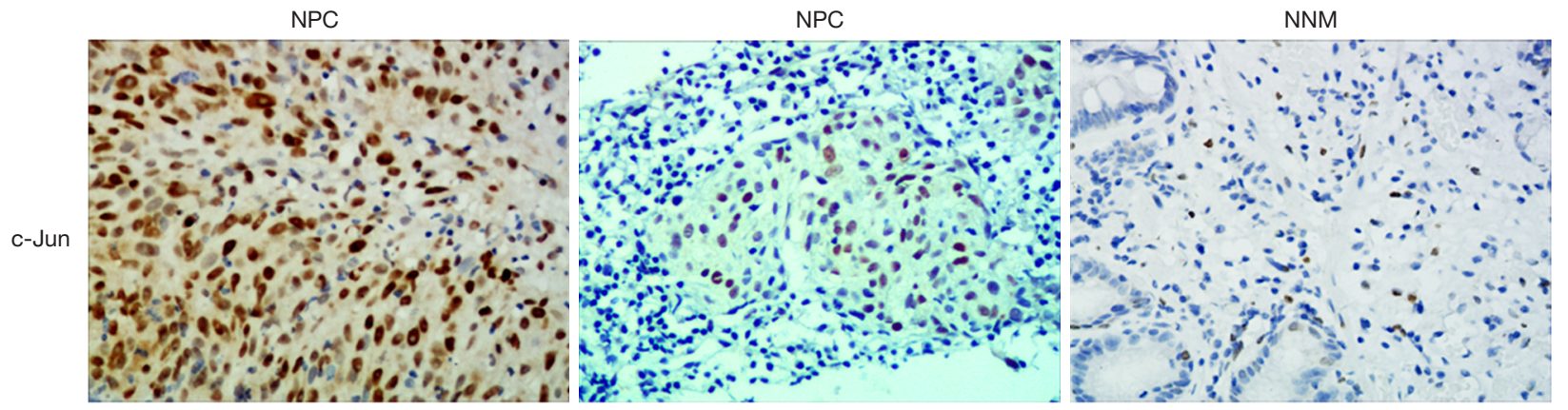

B

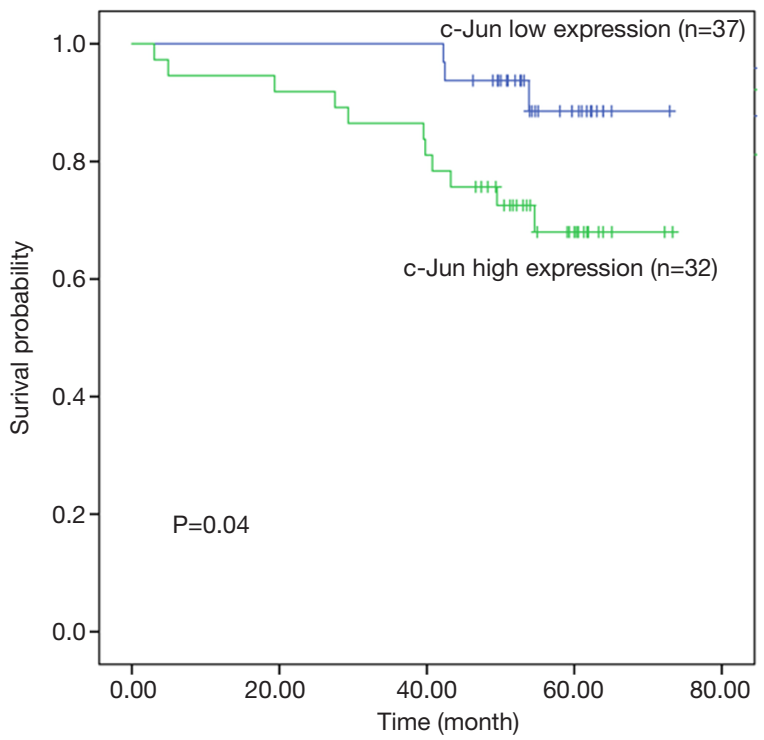

Figure 1 High expression of c-Jun was correlated with shorter overall survival of NPC patients. (A) NPC tissue with nuclear positivity and corresponding negative staining for c-Jun and NNM staining for c-Jun (magnification $\times 200$ ). (B) Kaplan-Meier survival curves illustrating survival time differences in patients with high expression $(\mathrm{n}=37)$ and low expression of nuclear c-Jun $(\mathrm{n}=32)$. NPC, nasopharyngeal carcinoma; NNM, normal nasopharyngeal mucosa.

Table 1 Expression of c-Jun in NPC and nasopharyngeal mucosa

\begin{tabular}{ccccc}
\hline \multirow{2}{*}{ Groups } & $\mathrm{N}$ & \multicolumn{2}{c}{ c-Jun, $\mathrm{n}(\%)$} & \multirow{2}{*}{$\mathrm{P}$ value } \\
\cline { 3 - 4 } & & High expression & Low expression & \\
\hline Cancer & 69 & $37(53.6)$ & $32(46.4)$ & \\
Normal & 21 & $4(19.0)$ & $17(81.0)$ & $<0.01$ \\
\hline
\end{tabular}

NPC, nasopharyngeal carcinoma.

patients (HR: 3.674, 95\% CI: 1.024-13.178, P<0.05), and multivariate Cox analysis showed that the expression of c-Jun was an independent factor for poor prognosis in NPC patients (Table 3) [hazard ratio (HR): 5.13; 95\% confidence interval (CI): $1.138-23.120 ; \mathrm{P}<0.05]$. TNM stage had no correlation with the prognosis of NPC patients $(\mathrm{P}>0.05)$.

\section{c-fun targeted PI3K/AKT in NPC}

To ascertain whether the PI3K/AKT/mTOR pathway can be regulated by c-Jun, the Sabiosciences website was used for prediction. Sequence analysis revealed c-Jun binding sites in both the PI3KCG and AKT1 promoters. PI3KCG and AKT1 promoter luciferase constructs were cotransfected with TFS-c-Jun into CNE-2R cells. A dual luciferase gene reporter system indicated that luciferase activity was significantly upregulated by TFSc-Jun in the (p-PI3KCG + TFS-Jun) cotransfection group and (p-AKT1 + TFS-c-Jun) cotransfection group (Figure 2A,B,C). c-Jun transactivated PI3KCG and AKT1 promoter activity. These results demonstrated that c-Jun directly targeted PI3K/AKT. 


\section{Silencing of c-fun inbibited autophagy activation by activating PI3K/AKT/mTOR patbway}

Since c-Jun was highly expressed in NPC tissues and

Table 2 Patient characteristics of 69 patients with NPC

\begin{tabular}{|c|c|c|c|c|}
\hline \multirow{2}{*}{$\begin{array}{l}\text { Clinicopathological } \\
\text { feature }\end{array}$} & \multirow[b]{2}{*}{$\mathrm{N}$} & \multicolumn{2}{|c|}{ C-Jun } & \multirow[b]{2}{*}{$P$ value } \\
\hline & & expression expression & $\begin{array}{l}\text { High } \\
\text { pression }\end{array}$ & \\
\hline Pathologic type & & & & 0.677 \\
\hline Differentiated & 4 & 1 & 3 & \\
\hline Undifferentiated & 65 & 31 & 34 & \\
\hline Gender & & & & 0.885 \\
\hline Male & 50 & 19 & 31 & \\
\hline Female & 19 & 13 & 6 & \\
\hline Age $(y)$ & & & & 0.007 \\
\hline$<50$ & 38 & 18 & 20 & \\
\hline$\geq 50$ & 31 & 24 & 17 & \\
\hline T stage & & & & 0.891 \\
\hline $\mathrm{T} 1-2$ & 13 & 8 & 5 & \\
\hline T3-4 & 56 & 34 & 32 & \\
\hline $\mathrm{N}$ stage & & & & 0.2 \\
\hline No & 2 & 1 & 1 & \\
\hline $\mathrm{N} 1-3$ & 67 & 31 & 36 & \\
\hline Clinical stage & & & & 0.889 \\
\hline II & 6 & 3 & 3 & \\
\hline III-IV & 63 & 29 & 34 & \\
\hline
\end{tabular}

NPC, nasopharyngeal carcinoma. correlated with the prognosis of NPC, we then conducted in vitro and in vivo functional and mechanistic experiments to explore whether c-Jun was associated with autophagy and radioresistance. The expression of c-Jun and autophagy activity were analyzed using western blotting. c-Jun was significantly decreased in CNE-2R cells with lentiviralmediated gene silencing technology (Figure $3 A, B, C$ ). The expression of LC3-II was decreased and P62 was upregulated after c-Jun silencing (Figure 4A,B), indicating that the level of autophagy was inhibited.

We further explored whether c-Jun targets PI3K/AKT. Our results showed that knockdown of c-Jun in CNE$2 \mathrm{R}$ cells inhibited the expression of phosphorylated PI3K (p110 $\gamma$ ), AKT, and mTOR, but total PI3K, AKT, and mTOR protein levels remained unchanged (Figure 4C,D). These results demonstrated that c-Jun could negatively regulate the expression of $\mathrm{PI} 3 \mathrm{~K}, \mathrm{AKT}$ and $\mathrm{mTOR}$ in $\mathrm{CNE}$ $2 \mathrm{R}$ cells.

To explore the role of PI3K/AKT in the regulation of mTOR, the inhibitor LY294002 was used to suppress the expression of phosphorylated PI3K in CNE-2R cells, and the results revealed that the expression of the phosphorylated proteins PI3K, AKT, and mTOR was simultaneously reduced (Figure $4 E, F$ ). These results indicated that mTOR was downregulated by suppressing PI3K. In addition, after downregulating PI3K, the expression of LC3-II was upregulated, while the expression of P62 was downregulated, indicating that the autophagy level was enhanced when PI3K was inhibited.

To further verify that mTOR is a downstream target of PI3K/AKT, RAPA, a specific inhibitor of mTOR, was applied to inhibit the expression of phosphorylated mTOR. No significant difference was observed among the groups in the expression of the phosphorylated forms of PI3K and

Table 3 Multivariate analysis of prognosis

\begin{tabular}{lccc}
\hline Factors & HR & $95 \% \mathrm{Cl}$ & $\mathrm{P}$ value \\
\hline C-Jun expression & 5.130 & $(1.138-23.10)$ & 0.033 \\
Age & 7.713 & $(1.714-29.516)$ & 0.892 \\
Gender & 0.909 & $(0.229-3.614)$ & 0.892 \\
Pathologic type & 0.389 & $(0.035-4.306)$ & 0.441 \\
T stage & 1.336 & $(0.163-10.976)$ & 0.787 \\
N stage & 0.125 & $(0.010-1.640)$ & 0.113 \\
Clinical stage & 1.064 & $(0.062-18.312)$ & 0.966 \\
\hline
\end{tabular}

$\mathrm{HR}$, hazard ratio; $95 \% \mathrm{Cl}$, 95\% confidence interval. 
A
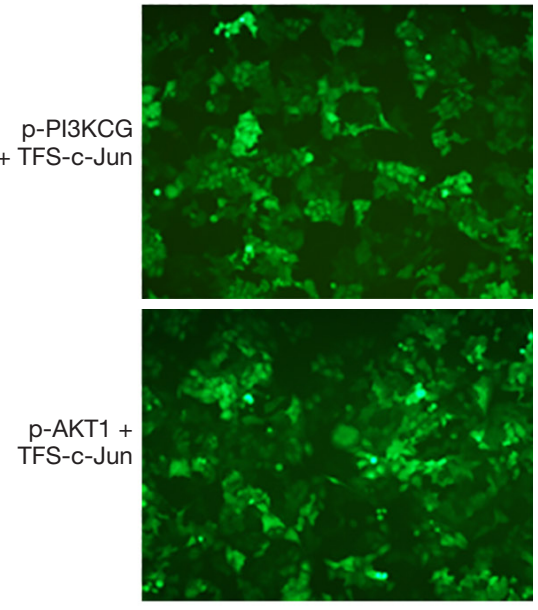
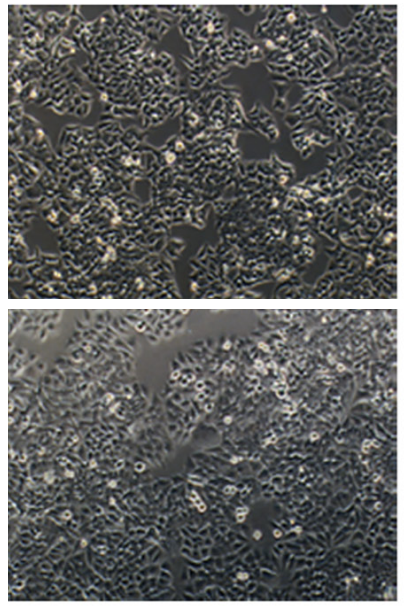

B

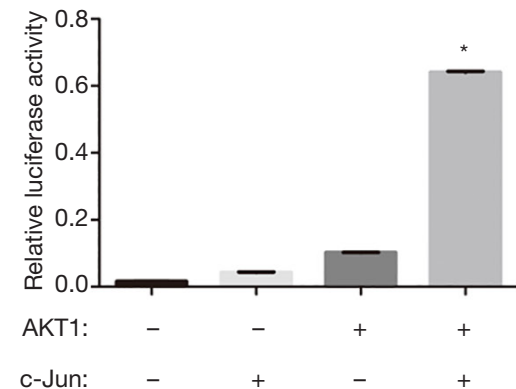

C

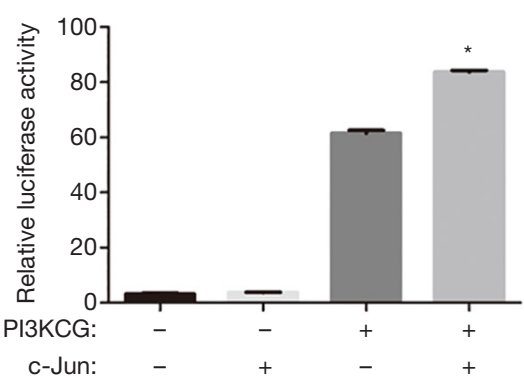

Figure 2 Dual-luciferase reporter assay verified that c-Jun targeted PI3K/AKT. (A) CNE-2R cells cotransfected with the PI3KCG or AKT1 promoter construct and TFS-c-Jun were observed by inverted fluorescence microscopy (magnification $\times 100$ ). (B,C) CNE-2R cells were cotransfected with c-Jun and different PI3KCG or AKT1 promoter-driven luciferase plasmids for 48 hours, and luciferase activity was determined by a luciferase reporter assay kit $\left({ }^{*}, \mathrm{P}<0.05\right.$ compared with the control).

A

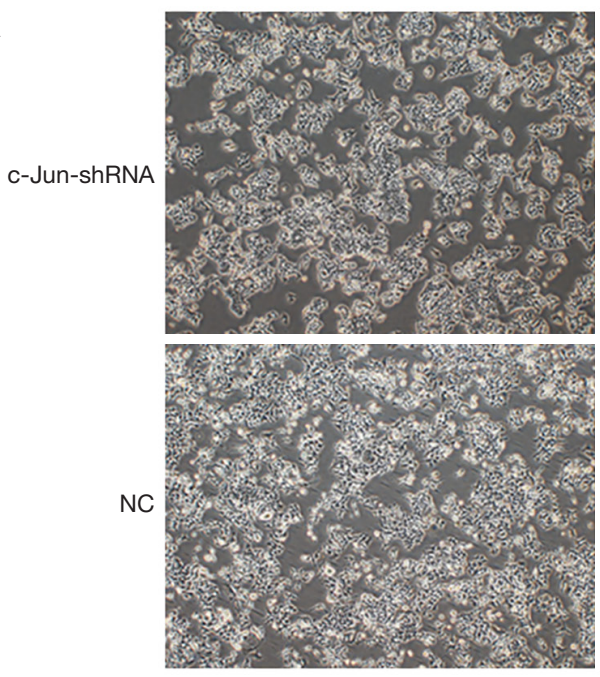

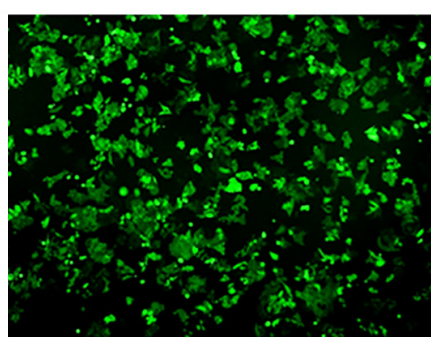

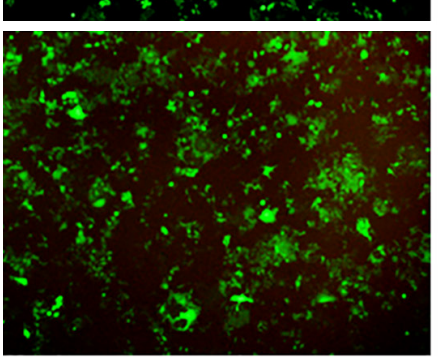

$B$

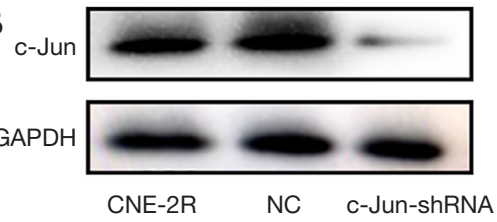

C

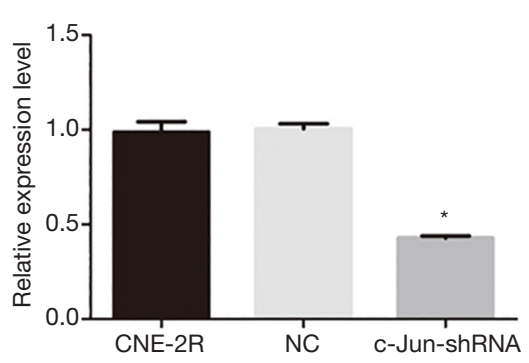

Figure 3 A stable silencing c-Jun CNE-2R model was established. (A) The transfection rate of lentivirus was observed by inverted fluorescence microscopy (magnification $\times 100$ ). (B) c-Jun expression was detected by western blotting. (C) The relative protein expression of each group ( ${ }^{*}, \mathrm{P}<0.05$ compared with control). mRNA, messenger RNA; NC, negative control; shRNA, short hairpin RNA.

AKT (Figure 4G,H). In addition, after inhibiting mTOR, the expression of the autophagy-related protein LC3-II was upregulated, and the expression of P62 was downregulated, suggesting that autophagy was enhanced when mTOR was inhibited.

In summary, these results demonstrated that c-Jun knockdown activated the PI3K/AKT/mTOR signaling pathway, thereby promoting autophagy in CNE-2R cells. 
A

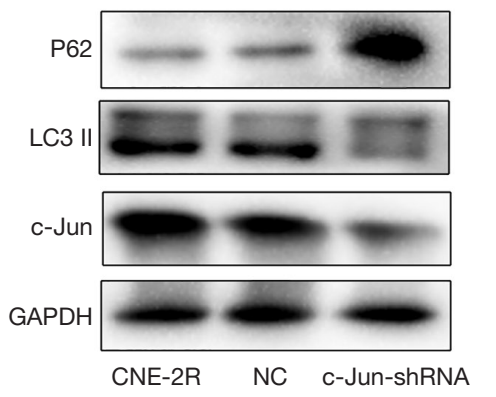

C

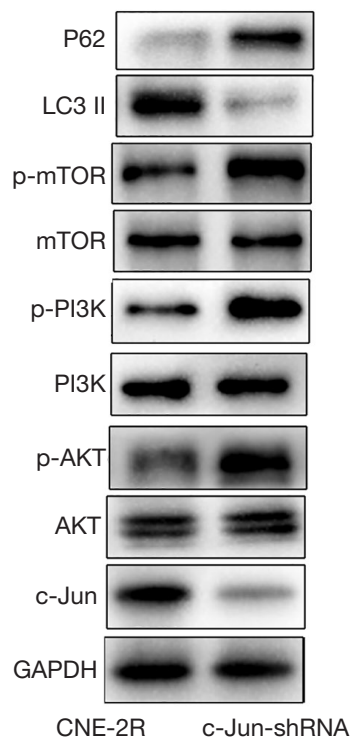

D

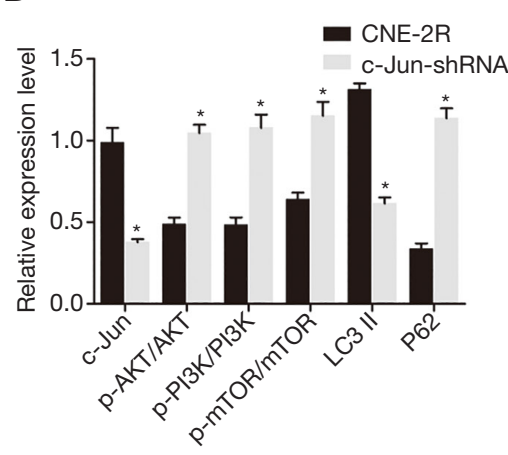

F
B

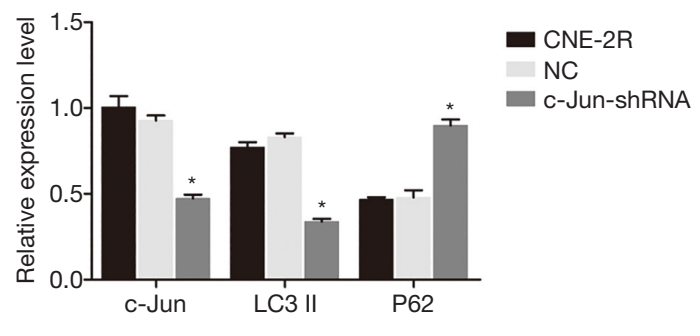

E

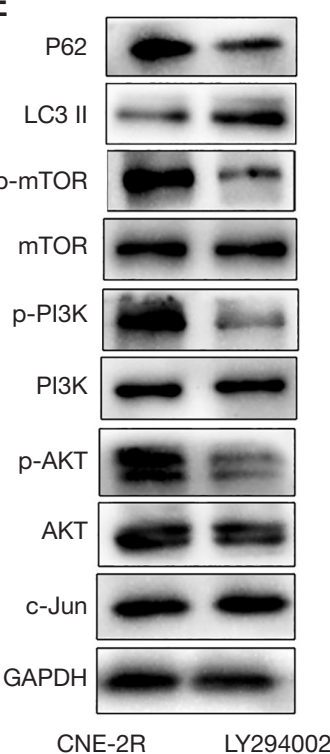

G

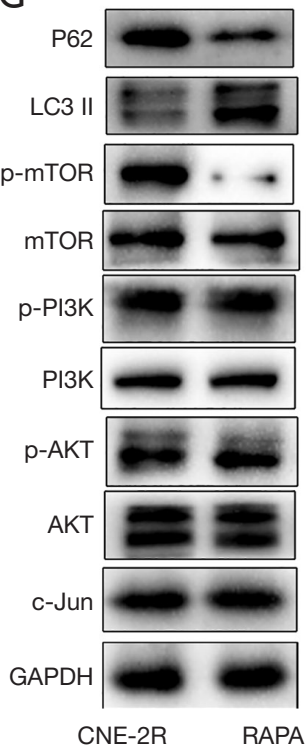

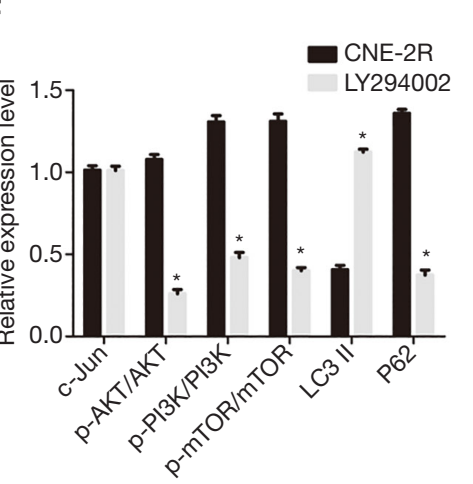

$\mathrm{H}$

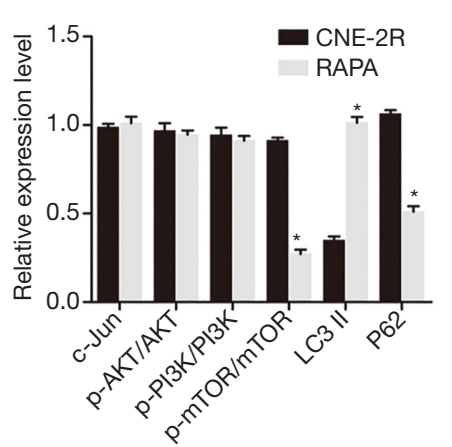

Figure 4 c-Jun regulates the PI3K/AKT/mTOR pathway to mediate autophagy in vitro. (A) Expression of LC3-II and P62 proteins was detected by western blotting after c-Jun silencing. (B) The relative protein expression of each group $\left({ }^{*}, \mathrm{P}<0.05\right.$ compared with the control). (C,F) Expression of LC3-II, P62, total AKT, PI3K, and mTOR protein and phosphorylation levels of AKT, PI3K, and mTOR after silencing c-Jun (*, P<0.05 compared with CNE-2R cells). (D,G) Expression of c-Jun, LC3-II, and P62 and phosphorylation levels of AKT, PI3K, and mTOR protein after treatment with the indicated concentration of the PI3K inhibitor (LY294002) for 6 hours (*, $\mathrm{P}<0.05$ compared with control). (E,H) Expression of c-Jun, LC3-II, P62, phosphorylated AKT, PI3K, and mTOR protein at the indicated concentration of the mTOR inhibitor (RAPA) for 6 hours $\left({ }^{*}, \mathrm{P}<0.05\right.$ compared with control). 


\section{c-7un knockdown inhibited NPC progression in vivo}

To further investigate the effect of c-Jun on NPC growth and radioresistance in vivo, NPC cells were injected into nude mice subcutaneously to construct a transplanted tumor model, and the tumors were irradiated when grown to $0.8-1 \mathrm{~cm}^{3}$. To further verify the effect of c-Jun silencing on sensitivity to radiation, RT was performed on nude mice 14 and 21 days after the injection of tumor cells. We found that the c-Jun knockdown group (c-Jun-shRNA + IR) and activating PI3K group (IGF-1 + IR) showed markedly decreased tumor volume growth compared with the control group (CNE-2R + IR). In contrast, inhibiting PI3K signaling via LY294004 and mTOR via RAPA promoted tumor volume growth (Figure $5 A, B, C$ ). These results suggested that silencing c-Jun and PI $3 \mathrm{~K}$ activation produced a more sensitive response to RT in xenografts than in the control group.

\section{Irradiation induced autophagy activation and upregulated c-fun expression in nude mouse-transplanted tumors}

After 10 Gy irradiation, P62 expression was downregulated, while LC3-II expression was upregulated (Figure 5D,E), indicating that the autophagy level was enhanced. Moreover, we found that c-Jun expression was upregulated after 10 Gy irradiation even though c-Jun was stably knocked down (Figure $5 D, E$ ). These results suggested that radiation could activate c-Jun and promote autophagy.

\section{c-fun knockdown inhibited autophagy and enhanced radiosensitivity by activating the PI3K/AKT/mTOR patbway in vivo}

To further identify whether c-Jun regulated the PI3K/ AKT/mTOR pathway in vivo, western blotting assays were applied to detect the effects of c-Jun knockdown on the expression of pathway-associated proteins, p-PI3K, p-AKT, p-mTOR, and P62 expression was upregulated, while LC3II expression was downregulated in the c-Jun knockdown group (c-Jun-shRNA + IR) and PI3K activated group (IGF-1 $+\mathrm{IR})$ compared to the control irradiated group (CNE-2R + IR) in the xenografts (Figure 5F,G,H). Moreover, CNE-2R cells treated with PI3K inhibitor (LY294002 + IR) attenuated the protein levels of p-PI3K, p-AKT, p-mTOR, and P62 while enhancing LC3-II expression compared to the control irradiated group (CNE-2R + IR). The mTOR-inhibited group (RAPA + IR) was not significantly different from the control irradiated group in terms of the expression of $\mathrm{p}-\mathrm{PI} 3 \mathrm{~K}$ and $\mathrm{p}-\mathrm{AKT}$. These results indicate that the PI3K/AKT/mTOR pathway may be involved in c-Jun-mediated growth promotion of NPC. mTORmediated autophagy was closely dependent on upstream PI3K/AKT signaling. These results demonstrated that the $\mathrm{PI} 3 \mathrm{~K} / \mathrm{AKT} / \mathrm{mTOR}$ signaling pathway could be activated by c-Jun downregulation. Autophagy activity was inhibited by decreased mTOR activity, resulting in radiosensitization in vivo.

\section{Discussion}

In the present study, we confirmed that c-Jun expression was significantly upregulated in NPC tissues in one independent cohort of NPC patients. c-Jun overexpression was correlated with worse NPC prognoses. Multivariate Cox analysis revealed that c-Jun expression was an independent risk factor for shorter overall survival in NPC. In addition, these patient characteristics illustrated that c-Jun can be used as a promising therapeutic target for NPC.

RT is the principal effective treatment modality for NPC, causing DNA double-stranded breaks (DSBs) by high-energy ionizing radiation that induces many types of cell death, including apoptosis, necrosis, and mitochondrial disorder (19). In addition to direct DNA damage, ionizing radiation damages DNA indirectly through the production of free radicals and reactive oxygen species. Radioresistance can be regarded as a process by which tumor cells adapt to radiation damage and resist ionizing radiation (20). Radioresistance is a process involving complicated mechanisms and changes in cellular function, such as cell cycle arrest, oncogene and tumor suppressor gene alteration, autophagy activation, and DNA damage repair mechanisms. During RT, surviving tumor cells can accelerate reproliferation and induce tumor recurrence and distant metastases (21). Increasing evidence has demonstrated that c-Jun, as an oncogene, plays a critical role in cell proliferation, apoptosis, and transformation and functions to control various cellular processes (22). Nevertheless, reports concerning the correlation between c-Jun and radioresistance in tumors are rare. Our previous study showed that the radioresistant CNE-2R cell line had higher levels of c-Jun than did radiosensitive parental cells (17), and that c-Jun was involved in radioresistance in NPC.

The PI3K/AKT/mTOR signaling pathway is one of the most frequently activated pathways in cancers. Recently, research has revealed that the PI3K/AKT/mTOR signaling pathway plays a crucial role in mediating multiple cellular 
A

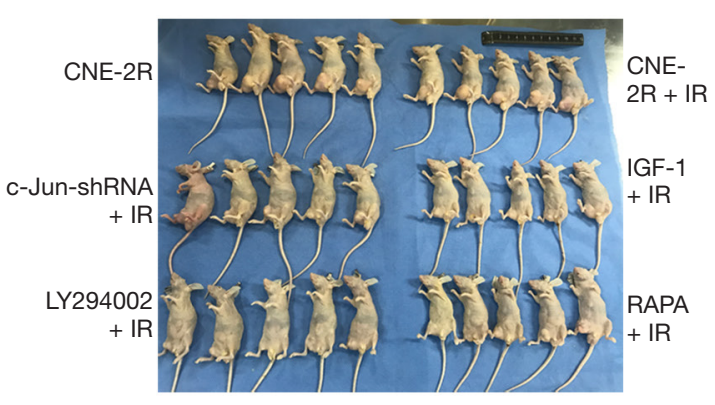

D

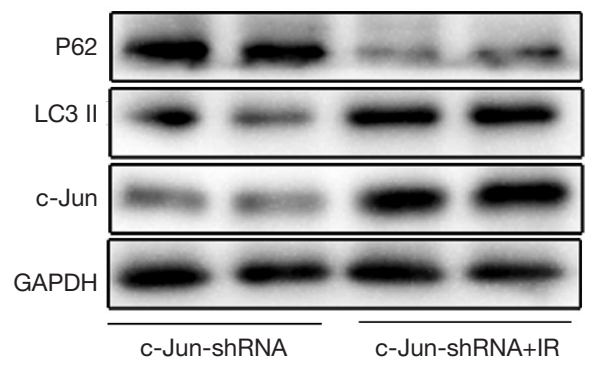

F

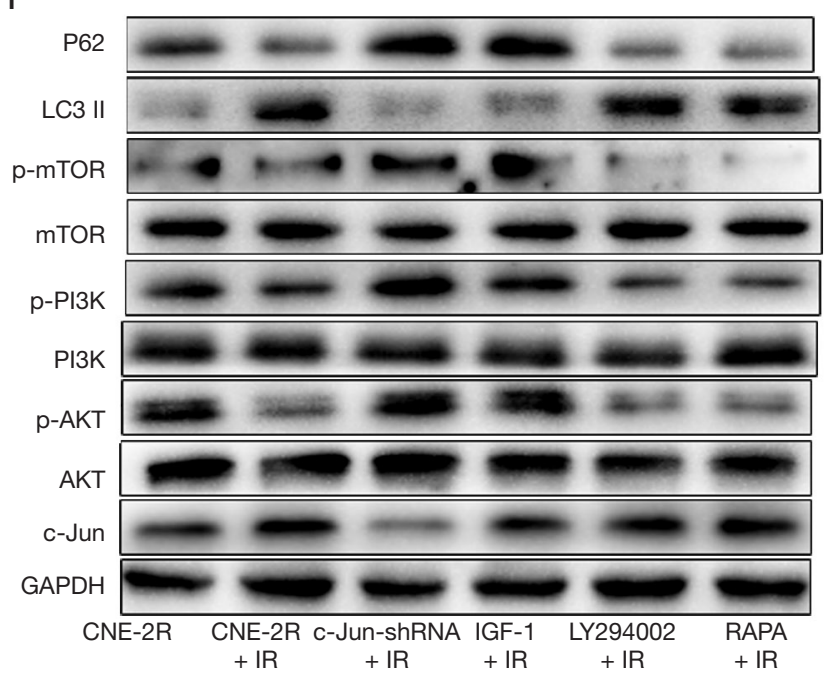

B

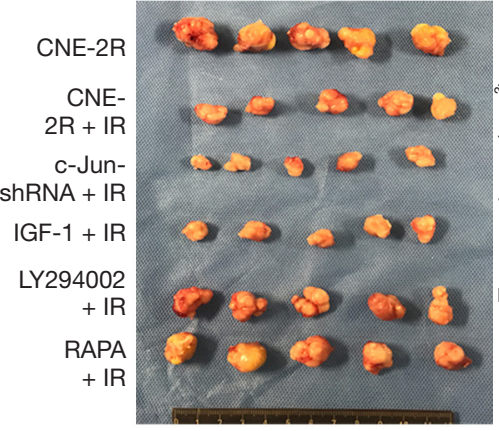

E

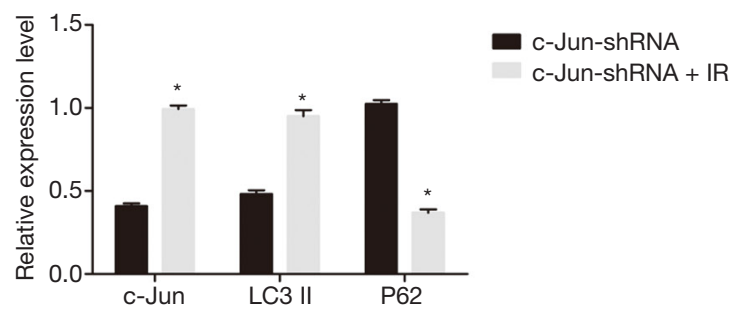

G

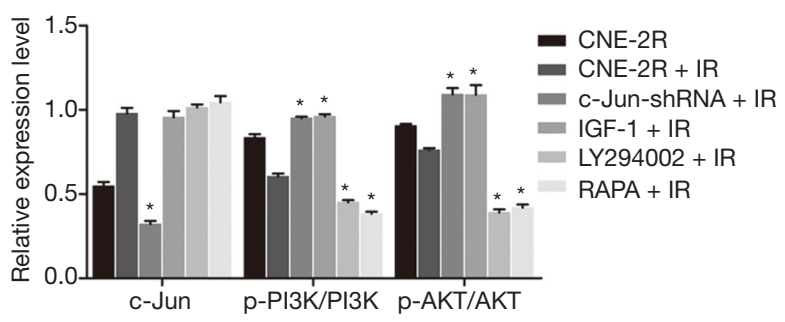

$\mathrm{H}$

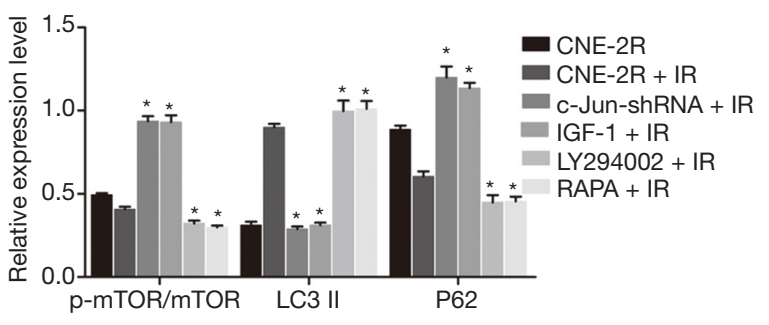

Figure 5 c-Jun mediates PI3K/AKT/mTOR pathway-induced radioresistance in vivo. (A,B) CNE-2R cells stably downregulating c-Jun or control were injected subcutaneously into nude mice. The mice were irradiated with a dose of 0 or 5 Gy at day 14 and 20 . (C) The tumor volumes were measured every 3 days in each group (*, $\mathrm{P}<0.05)$. (D,E) Expression of c-Jun, LC3-II and P62 was increased after c-Jun downregulation with or without $10 \mathrm{~Gy}$ irradiation in transplanted nude mouse tumors $\left.{ }^{*}, \mathrm{P}<0.05\right)$. (F,G,H) Downregulating c-Jun activated the pathway axis and decreased autophagy activation. LY294002 or RAPA inhibitors were used to inhibit the PI3K/AKT/mTOR pathway, and autophagy activation of transplanted tumors was enhanced $\left({ }^{*}, \mathrm{P}<0.05\right.$ compared with control).

processes, including cell growth, proliferation, metabolism, and chemoradiotherapy resistance (23). Therefore, we hypothesized that c-Jun participates in the regulation of PI3K/AKT/mTOR. A dual-luciferase reporter system was used to prove that c-Jun was involved in the regulation of the promoter region of PI3K/AKT. We then detected the expression of the proteins in this pathway by knocking down c-Jun in the CNE-2R cell line and found that the 
$\mathrm{PI} 3 \mathrm{~K} / \mathrm{AKT} / \mathrm{mTOR}$ signaling pathway was activated. The downregulation of c-Jun increased the phosphorylation of pathway-related proteins, indicating that silencing c-Jun in CNE-2R cells could activate the PI3K/AKT/mTOR signaling pathway.

Autophagy provides nutrients for tumor cells, thus promoting tumor growth, and autophagy inhibition has been proposed as a cancer therapy strategy. Our previous study demonstrated that autophagy could serve as a selfprotective mechanism and promote the survival of NPC cells during radiation (10 Gy) (24). Other studies have shown that autophagy is related to radioresistance in prostate cancer, lung cancer, esophageal cancer, and colorectal cancer (25-27). In addition, the $\mathrm{PI} 3 \mathrm{~K} / \mathrm{AKT} / \mathrm{mTOR}$ signaling pathway is regarded as one of the main pathways involved in the initiation and regulation of autophagy (28). We found that after applying ionizing radiation in NPC cells with c-Jun knockdown, the autophagy level was decreased. IGF-1 has been recognized as an autophagy inhibitor that can promote cell death by activating the PI3K/AKT/mTOR signaling pathway (29). We found that PI3K/AKT/mTOR pathway activation additionally caused autophagy suppression, but the underlying mechanisms need to be further elucidated.

In conclusion, our study showed that c-Jun promoted NPC growth by targeting the PI3K/AKT/mTOR pathway. We further found that c-Jun enhanced autophagy, which promoted radioresistance in CNE-2R cells. Silencing c-Jun combined with RT enhanced the radiosensitivity of NPC and improved therapeutic efficacy.

\section{Conclusions}

Taken together, our data demonstrated that c-Jun overexpression was closely related to the poor prognosis of NPC, suggesting that it may be a promising biomarker for predicting the prognosis of NPC. c-Jun could activate autophagy and mediate radioresistance via the PI3K/AKT/ mTOR pathway. In addition, targeting the c-Jun and PI3K/ AKT/mTOR pathways may provide potential strategies for the treatment of radioresistant NPC.

\section{Acknowledgments}

We thank Dr. Shaohua Chen from Guangxi Medical University for his instructional advice on the revision of the manuscript.

Funding: This work was supported by grants from the National Natural Science Foundation of China
(No. 81760544), the Scientific Research \& Technical Development Project of Wuming District, Nanning City (No. 20200214), the Key Laboratory of High-IncidenceTumor Prevention and Treatment, Independent Research Project (No. GKE-ZZ202014).

\section{Footnote}

Reporting Checklist: The authors have completed the REMARK reporting checklist. Available at https://dx.doi. org/10.21037/atm-21-2563

Data Sharing Statement: Available at https://dx.doi. org/10.21037/atm-21-2563

Conflicts of Interest: All authors have completed the ICMJE uniform disclosure form (available at https://dx.doi. org/10.21037/atm-21-2563). The authors have no conflicts of interest to declare.

Ethical Statement: The authors are accountable for all aspects of the work in ensuring that questions related to the accuracy or integrity of any part of the work are appropriately investigated and resolved. All procedures performed in this study involving human participants were in accordance with the Declaration of Helsinki (as revised in 2013). The study was approved by Research Ethics Committee of Guangxi Medical University Cancer Hospital and informed consent was taken from all the patients. Experiments were performed under a project license granted by the Laboratory Animal Care and Use Committee of Guangxi Medical University Cancer Hospital, in compliance with Guangxi Medical University Cancer Hospital guidelines for the care and use of animals.

Open Access Statement: This is an Open Access article distributed in accordance with the Creative Commons Attribution-NonCommercial-NoDerivs 4.0 International License (CC BY-NC-ND 4.0), which permits the noncommercial replication and distribution of the article with the strict proviso that no changes or edits are made and the original work is properly cited (including links to both the formal publication through the relevant DOI and the license). See: https://creativecommons.org/licenses/by-nc-nd/4.0/.

\section{References}

1. Ji MF, Sheng W, Cheng WM, et al. Incidence and 
mortality of nasopharyngeal carcinoma: interim analysis of a cluster randomized controlled screening trial (PRONPC-001) in southern China. Ann Oncol 2019;30:1630-7.

2. Sun X, Su S, Chen C, et al. Long-term outcomes of intensity-modulated radiotherapy for 868 patients with nasopharyngeal carcinoma: an analysis of survival and treatment toxicities. Radiother Oncol 2014;110:398-403.

3. Di M, Wang M, Miao J, et al. CHAF1B induces radioresistance by promoting DNA damage repair in nasopharyngeal carcinoma. Biomed Pharmacother 2020;123:109748.

4. Shaulian E, Karin M. AP-1 in cell proliferation and survival. Oncogene 2001;20:2390-400.

5. Yu X, Chen D, Wang L, et al. Wogonoside inhibits inflammatory cytokine production in lipopolysaccharidestimulated macrophage by suppressing the activation of the JNK/c-Jun signaling pathway. Ann Transl Med 2020;8:532.

6. Qi S, Kim DJ, Stjepanovic G, et al. Structure of the human Atg13-Atg101 HORMA heterodimer: an interaction hub within the ULK1 complex. Structure 2015;23:1848-57.

7. Klein SR, Piya S, Lu Z, et al. C-Jun N-terminal kinases are required for oncolytic adenovirus-mediated autophagy. Oncogene 2015;34:5295-301.

8. Zhu X, Zhou M, Liu G, et al. Autophagy activated by the c-Jun N-terminal kinase-mediated pathway protects human prostate cancer PC3 cells from celecoxib-induced apoptosis. Exp Ther Med 2017;13:2348-54.

9. Li Z, Zhang L, Gao M, et al. Endoplasmic reticulum stress triggers Xanthoangelol-induced protective autophagy via activation of JNK/c-Jun Axis in hepatocellular carcinoma. J Exp Clin Cancer Res 2019;38:8. Retraction in: J Exp Clin Cancer Res 2020;39:164.

10. Gao Y, Qi W, Liu S, et al. Acid-induced autophagy protects human gastric cancer cells from apoptosis by activating Erk1/2 pathway. Transl Cancer Res 2019;8:1560-70.

11. Boya P, González-Polo RA, Casares N, et al. Inhibition of macroautophagy triggers apoptosis. Mol Cell Biol 2005;25:1025-40.

12. Digomann D, Linge A, Dubrovska A. SLC3A2/CD98hc, autophagy and tumor radioresistance: a link confirmed. Autophagy 2019;15:1850-1.

13. Chan AT. Current treatment of nasopharyngeal carcinoma. Eur J Cancer 2011;47 Suppl 3:S302-3.

14. Lai CH, Chen MF, Fang FM, et al. Estimation of life expectancy and quality-adjusted life expectancy in nonmetastatic nasopharyngeal cancer patients treated by intensity-modulated radiotherapy with or without chemotherapy. Oral Oncol 2014;50:646-50.

15. Chaurasia M, Bhatt AN, Das A, et al. Radiation-induced autophagy: mechanisms and consequences. Free Radic Res 2016;50:273-90.

16. Sasaki T. New guidelines to evaluate the response to treatment "RECIST". Gan To Kagaku Ryoho 2000;27:2179-84.

17. Guo Y, Zhu XD, Qu S, et al. Identification of genes involved in radioresistance of nasopharyngeal carcinoma by integrating gene ontology and protein-protein interaction networks. Int J Oncol 2012;40:85-92.

18. Guo SY, Zhu XD, Ge LY, et al. RNAi-mediated knockdown of the c-jun gene sensitizes radioresistant human nasopharyngeal carcinoma cell line $\mathrm{CNE}-2 \mathrm{R}$ to radiation. Oncol Rep 2015;33:1155-60.

19. Sgouros G. Alpha-particles for targeted therapy. Adv Drug Deliv Rev 2008;60:1402-6.

20. Srinivas US, Tan BWQ, Vellayappan BA, et al. ROS and the DNA damage response in cancer. Redox Biol 2019;25:101084.

21. Nijkamp MM, Hoogsteen IJ, Span PN, et al. Spatial relationship of phosphorylated epidermal growth factor receptor and activated AKT in head and neck squamous cell carcinoma. Radiother Oncol 2011;101:165-70.

22. Kielosto M, Nummela P, Katainen R, et al. Reversible regulation of the transformed phenotype of ornithine decarboxylase- and ras-overexpressing cells by dominantnegative mutants of c-Jun. Cancer Res 2004;64:3772-9.

23. Yu JS, Cui W. Proliferation, survival and metabolism: the role of PI3K/AKT/mTOR signalling in pluripotency and cell fate determination. Development 2016;143:3050-60.

24. Zhou ZR, Zhu XD, Zhao W, et al. Poly(ADP-ribose) polymerase- 1 regulates the mechanism of irradiationinduced CNE-2 human nasopharyngeal carcinoma cell autophagy and inhibition of autophagy contributes to the radiation sensitization of CNE-2 cells. Oncol Rep 2013;29:2498-506.

25. Karagounis IV, Kalamida D, Mitrakas A, et al. Repression of the autophagic response sensitises lung cancer cells to radiation and chemotherapy. Br J Cancer 2016;115:312-21.

26. Koukourakis MI, Kalamida D, Mitrakas A, et al. Intensified autophagy compromises the efficacy of radiotherapy against prostate cancer. Biochem Biophys Res Commun 2015;461:268-74.

27. Yang Y, Sun X, Yang Y, et al. Gambogic acid enhances the radiosensitivity of human esophageal cancer cells by inducing reactive oxygen species via targeting Akt/mTOR 
pathway. Tumour Biol 2016;37:1853-62.

28. Xu Z, Han X, Ou D, et al. Targeting PI3K/AKT/mTORmediated autophagy for tumor therapy. Appl Microbiol Biotechnol 2020;104:575-87.

29. Choi EJ, Cho BJ, Lee DJ, et al. Enhanced cytotoxic effect

Cite this article as: Sun Y, Chen K, Lin G, Wan F, Chen L, Zhu X. Silencing c-Jun inhibits autophagy and abrogates radioresistance in nasopharyngeal carcinoma by activating the PI3K/AKT/mTOR pathway. Ann Transl Med 2021;9(13):1085. doi: $10.21037 / \mathrm{atm}-21-2563$ of radiation and temozolomide in malignant glioma cells: targeting PI3K-AKT-mTOR signaling, HSP90 and histone deacetylases. BMC Cancer 2014;14:17.

(English Language Editor: J. Gray) 